\title{
Magnetic Investigations of Steel Degradation Using a Magnetic Hysteresis Scaling Technique
}

\author{
Satoru Kobayashi *, Kodai Miura, Yuki Narita and Seiki Takahashi \\ Faculty of Science and Engineering, Iwate University, Morioka 020-8551, Japan; chappy91@live.jp (K.M.); \\ yuki07231127@gmail.com (Y.N.); koba_ucsb@hotmail.co.jp (S.T.) \\ * Correspondence: koba@iwate-u.ac.jp; Tel.: +81-19-621-6350
}

Received: 11 November 2017; Accepted: 19 December 2017; Published: 21 December 2017

\begin{abstract}
We report the results of magnetic measurements on austenitic stainless steels and duplex stainless steels using a magnetic hysteresis scaling technique. Unlike saturation hysteresis loops, this scaling technique, which uses a set of minor hysteresis loops, can be used in low measurement fields. We show that there is a universal scaling power law between minor-loop parameters, which is independent from the level of deformation. The behavior of a coefficient deduced from the scaling law was explained from the viewpoint of the morphology of a ferromagnetic phase.
\end{abstract}

Keywords: magnetic hysteresis; austenitic stainless steel; duplex stainless steel; non-destructive evaluation

\section{Introduction}

Studies on magnetic hysteresis scaling rules started about one century ago due to increasing demand for electrical steels; with regards to this, accurate knowledge of power losses at high flux densities was particularly important. For bulk ferromagnetic steels, it is known that a hysteresis loss of flux-density-magnetic-field (B-H) loops, or $W_{\mathrm{F}}{ }^{*}$, is related by power law to the maximum flux density $\left(B_{\mathrm{a}}{ }^{*}\right)$, with a scaling exponent of $n_{\mathrm{F}}=1.6$ in the medium flux density range [1]. This is calculated using the following equation:

$$
W_{\mathrm{F}}^{*}=W_{\mathrm{F}}^{0}\left(B_{\mathrm{a}}{ }^{*} / B_{\mathrm{a}}\right)^{n_{\mathrm{F}}}
$$

where the structure-sensitive magnetic property $\left(W_{\mathrm{F}}^{0}\right)$ is a coefficient and $B_{\mathrm{a}}$ is a normalization constant. This relationship (known as the Steinmetz law) has long been used, but its use is restricted mainly to the field of electricity.

Recently, we performed magnetic investigations on ferromagnetic steels in which material properties, such as defect density, are systematically changed by plastic deformation, neutron irradiation, etc. [2-4]. We found that hysteresis scaling relations exist between other hysteresis parameters and that the scaling exponent is almost independent of external and internal conditions (e.g., temperature, stress, microstructure, defect density, etc.). A coefficient of the scaling is more sensitive to the defect density than the coercivity of the saturation loop; it is also proportionally related to mechanical and physical properties, such as Vickers hardness, yield stress, and ductile-brittle transition temperature. The magnetic method using magnetic hysteresis scaling is, therefore, a possible non-destructive evaluation (NDE) technique for materials degradation because of its sensitivity to lattice defects and its low measurement field.

In this article, we will review the results of our magnetic hysteresis scaling for materials characterization of various types of steels. Particular emphasis will be given to the magnetic evaluation of austenitic stainless steels in which ferromagnetic strain-induced $\alpha^{\prime}$-martensite particles are formed in a paramagnetic austenitic (fcc- $\gamma$ ) matrix due to either plastic deformation or subzero cooling. Recent results for duplex stainless steels, including ferromagnetic ferrite (bcc $\alpha$ ) phase and paramagnetic austenitic (fcc $\gamma$ ) phase, are also given. 


\section{Austenitic Stainless Steel}

Austenitic stainless steel is widely used for manufacturing power plant structural components, such as pipelines, because of its high ductility, high corrosion resistance, and high strength. Under certain power plant conditions, such as temperature cycling and cycling loading, fatigue degradation takes place. Degradation in 304 stainless steel is particularly associated with martensitic transformation from an austenitic $(\gamma)$ phase to a martensite $\left(\alpha^{\prime}\right)$ one. Therefore, the magnetic method can be a possible NDE technique to evaluate the degradation because $\alpha^{\prime}$-martensite is the only ferromagnetic phase in this steel.

Traditionally, initial permeability and saturation magnetization have been used to evaluate the quantity of $\alpha^{\prime}$-martensite [5]. Recently, though, it was reported that coercivity is useful to characterize aspects of morphology, such as size and distribution [6-10]. However, the measurement field is on the order of $1 \mathrm{MA} / \mathrm{m}$, and this high field is disadvantageous for the integrity assessment, which requires a compact device. In this study, we have developed the scaling analysis method of magnetic minor hysteresis loops to characterize ferromagnetic $\alpha^{\prime}$-martensite in 304 stainless steel subjected to plastic deformation $[11,12]$ and/or cooling at $77 \mathrm{~K}$. The universal scaling rule of minor loops and the magnetic coefficient, being sensitive to the morphology of $\alpha^{\prime}$-martensite, have been obtained and discussed in detail.

\subsection{Materials and Experiments}

The chemical composition of 304 stainless steel used in this study is noted in Table 1. Steel plates with a thickness of $5 \mathrm{~mm}$ were austenitized at $1050{ }^{\circ} \mathrm{C}$ for one hour, then given a water quench. The plate samples were then deformed up to a tensile stress of $545 \mathrm{MPa}$ at room temperature to induce the formation of ferromagnetic $\alpha^{\prime}$-martensites. Fourteen samples with different stresses were prepared.

Table 1. Chemical composition of 304 stainless steel.

\begin{tabular}{ccccccccc}
\hline Element & $\mathbf{C}$ & $\mathbf{S i}$ & $\mathbf{M n}$ & $\mathbf{P}$ & $\mathbf{S i}$ & $\mathbf{N i}$ & $\mathbf{C r}$ & $\mathbf{F e}$ \\
\hline wt. $\%$ & 0.088 & 0.480 & 1.630 & 0.023 & 0.001 & 8.200 & 18.700 & Bal. \\
\hline
\end{tabular}

For magnetic measurements, we prepared two samples for each type of stress: (1) a ring wound with 220-turn exciting and 120-turn pickup coils and, (2) small plates with the long axis parallel to the stress direction. The plates were used to determine the volume fraction of $\alpha^{\prime}$-martensite though saturation magnetization measurements from a superconducting quantum interference device (SQUID) magnetometer MPMS-XL (Quantum Design, San Diego, CA, USA). A saturation magnetization of $154 \mathrm{emu} / \mathrm{g}$ was assumed for a volume fraction of $100 \%$. As shown in Figure 1a, the volume fraction of $\alpha^{\prime}$-martensites increases with tensile stress and reaches $19.5 \%$ after plastic deformation with a maximum stress of $535 \mathrm{MPa}$. The martensite phase was confirmed with optical microscopy and transmission electron microscopy (TEM). 

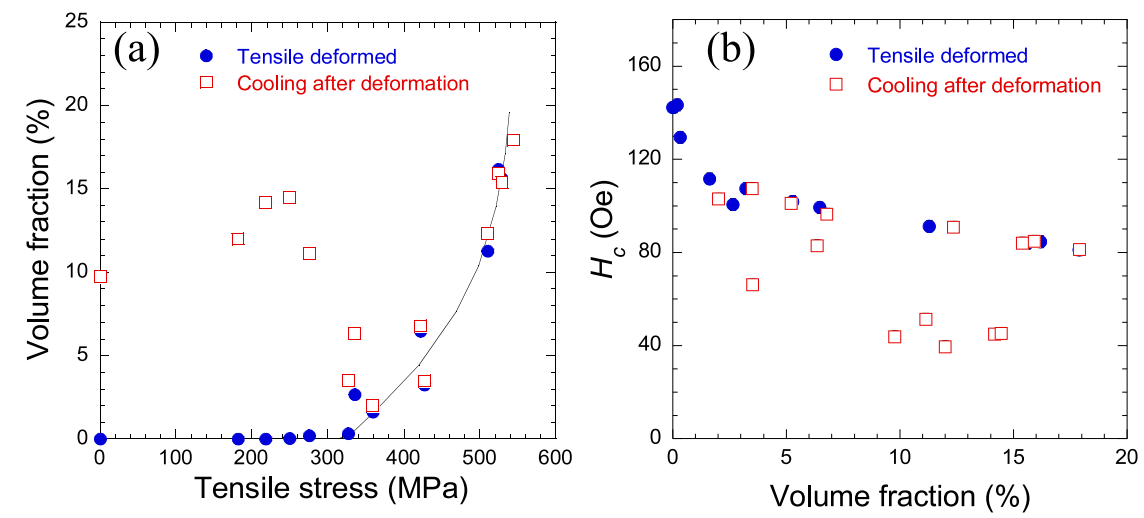

Figure 1. (a) Volume fraction of $\alpha^{\prime}$-martensites as a function of tensile stress. (b) Coercivity as a function of volume fraction. The solid and open symbols denote the data before and after cooling at $77 \mathrm{~K}$ for five minutes.

In Figure 2, a setup of our minor-loop measurement system is schematically shown. For the ring sample wound with both exciting and pickup coils, a set of magnetic minor hysteresis loops with various field amplitudes $\left(H_{\mathrm{a}}\right)$ up to $20 \mathrm{kA} / \mathrm{m}$ were measured using a fluxmeter. Triangular voltage with a frequency of $0.2 \mathrm{~Hz}$ (obtained from a function generator) was applied to a bipolar power supply. The bipolar power supply converts the voltage to current and amplifies it. The amplified current was then applied to the exciting coil to generate a cyclic magnetic field; this magnetized the sample. The magnetic field within the sample was obtained from the voltage across a 1-ohm resistance connected to the exciting coil in series. The induced voltage of the pickup coil was amplified with a gain of $60 \mathrm{~dB}$ and then purified by a low-pass filter with a cutoff frequency of $40 \mathrm{~Hz}$. After this signal processing, the induced voltage was integrated to obtain a magnetic flux within the sample. A set of minor hysteresis loops was measured by gradually increasing the amplitude of the wave current. The step of the increase of $H_{\mathrm{a}}$ was about $100 \mathrm{~A} / \mathrm{m}$. Before measuring each minor loop, the sample was demagnetized with a decaying alternating magnetic field with a frequency of $10 \mathrm{~Hz}$. These minor loops are different from the major hysteresis loops obtained with $H_{\mathrm{a}}$ that are sufficiently large for saturation. Figure 3a shows an example of a set of minor loops we measured.

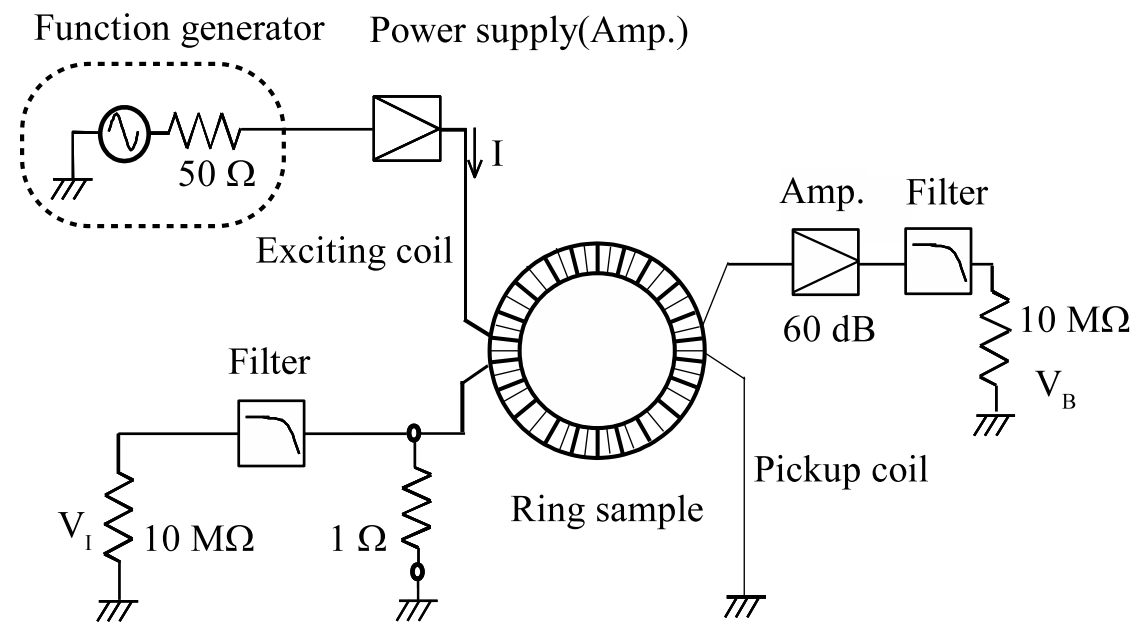

Figure 2. Schematic diagram of electric circuit for minor loop measurements.

To further investigate the effect of morphology on the minor-loop properties, the samples were subsequently cooled in a liquid nitrogen bath for five minutes. 

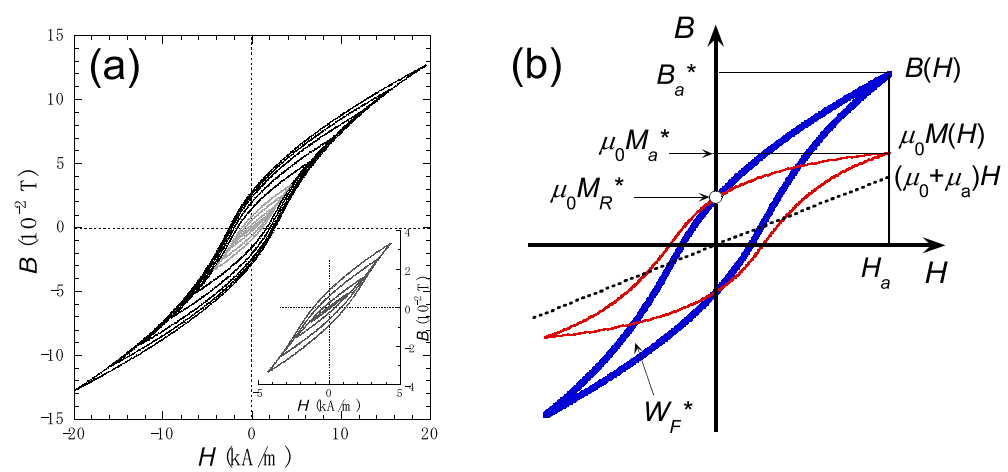

Figure 3. (a) A set of minor hysteresis loops. The data for $502 \mathrm{MPa}$ are given as an example. Minor loops with $H_{\mathrm{a}}$ below $4 \mathrm{kA} / \mathrm{m}$ (gray loops and the inset) were used to obtain minor-loop coefficient $\left(W_{\mathrm{m}}{ }^{0}\right)$. (b) Schematic diagram of the hysteresis loops and the parameters of the magnetic-flux-density $B(H)$ loop (blue thick line). In addition to $\mu_{0} M(H)$ (red thin line) due to ferromagnetic $\alpha^{\prime}$-martensites, both $\mu_{\mathrm{a}} H$ and $\mu_{0} H$ (dotted line) are involved in the $B(H)$ loop. Here, $M(H)$ is the magnetization, and $\mu_{0}$ and $\mu_{\mathrm{a}}$ denote the permeability of the vacuum and the austenitic matrix, respectively.

\subsection{Results and Discussion}

A set of minor hysteresis loops was analyzed according to the method developed for bulk soft ferromagnetic materials [2,3]. For each minor loop, the parameters such as $B_{\mathrm{a}}{ }^{*}$ and $W_{\mathrm{F}}{ }^{*}$ were obtained and the relationships between them examined. Note that samples with a volume fraction lower than $2 \%$ were not used for the analysis because of the very small signal from ferromagnetic martensites.

Figure 4a shows the double logarithmic plot of the relationship between $B_{\mathrm{a}}{ }^{*}$ and $W_{\mathrm{F}}{ }^{*}$ taken at various tensile stresses. Unlike usual soft ferromagnetic materials, it was found that the power law relationship does not hold true for ferromagnetic $\alpha^{\prime}$-martensites formed in an austenitic matrix. Since high magnetic fields are required to magnetize the $\alpha^{\prime}$-martensites, vacuum permeability due to the applied fields is considered to significantly contribute to the observed flux density. In addition, the paramagnetic permeability of an austenitic matrix also contributes because the volume fraction of ferromagnetic martensites is not so high. Therefore, the magnetic flux density, or $B(H)$, is no longer approximated by $\mu_{0} M(H)$ due to the sample magnetization; $B(H)$ would be given by a summation of $\mu_{0} M(H), \mu_{\mathrm{a}} H$, and $\mu_{0} H$ due to the ferromagnetic $\alpha^{\prime}$-martensites, austenitic matrix, and applied field, respectively, as shown in Figure $3 \mathrm{~b}$. Here, $\mu_{0}$ is the vacuum permeability and $\mu_{\mathrm{a}}$ is the paramagnetic permeability of the austenitic matrix. Consequently, the properties of the $B(H)$ loop such as $B_{\mathrm{a}}{ }^{*}$ shown in Figure $3 \mathrm{~b}$ is largely different from the corresponding maximum magnetization $\left(M_{\mathrm{a}}{ }^{*}\right)$ in the $\mu_{0} M(H)$ loop. This is the reason why the scaling rule used for soft ferromagnetic materials does not hold true for ferromagnetic $\alpha^{\prime}$-martensites formed in an austenitic matrix.

On the other hand, the parameters remanence $\left(M_{\mathrm{R}}{ }^{*}\right)$ and $W_{\mathrm{F}}{ }^{*}$ are independent of this contribution, and magnetic property obtained from the relationship may give useful information on $\alpha^{\prime}$-martensite. Therefore, a magnetic property obtained from the relationship between these two parameters may reveal intrinsic features of $\alpha^{\prime}$-martensites.

Figure $4 \mathrm{~b}$ shows the relationship plotted on a double logarithmic scale for various levels of tensile stress. One clearly sees that the $W_{\mathrm{F}}{ }^{*}-M_{\mathrm{R}}{ }^{*}$ curves exhibit straight lines over a wide range of $M_{\mathrm{R}}{ }^{*}$ and that the slope is almost independent of the volume fraction. This linearity strongly indicates the presence of a scaling power law rule between $W_{\mathrm{F}}{ }^{*}$ and $M_{\mathrm{R}}{ }^{*}$. In order to extract the intrinsic magnetic properties of $\alpha^{\prime}$-martensites, we assume a new equation, given as:

$$
W_{\mathrm{F}}{ }^{*}=W_{\mathrm{m}}^{0}\left(B_{\mathrm{R}}{ }^{*} / B_{\mathrm{a}}\right)^{n_{\mathrm{m}}}
$$

where $W_{\mathrm{m}}{ }^{0}$ and $n_{\mathrm{m}}$ are a coefficient and power law exponent, respectively, and $B_{\mathrm{R}}{ }^{*}$ is the remanent flux density of a minor loop. The normalization constant was set to 1 in this study. Least-squares 
fits to Equation (2) yielded an almost constant value of $n_{\mathrm{m}}=1.40 \pm 0.04$. Here, minor loops with $H_{\mathrm{a}}$ below $4 \mathrm{kA} / \mathrm{m}$ were used for the fits (Figure 3a). This value is independent of the volume fraction of $\alpha^{\prime}$-martensites. Figure 5a shows the minor-loop coefficient as a function of volume fraction. For the strain-induced $\alpha^{\prime}$-martensite, the coefficient monotonically decreases with the volume fraction; this is consistent with the behavior of coercivity, as shown in Figure 1b. This reflects the increase of martensite size alongside the volume fraction; this is because the pinning strength of magnetic domain walls at the $\alpha^{\prime}-\gamma$ phase boundary weakens with the increasing volume-surface ratio $[9,10]$.
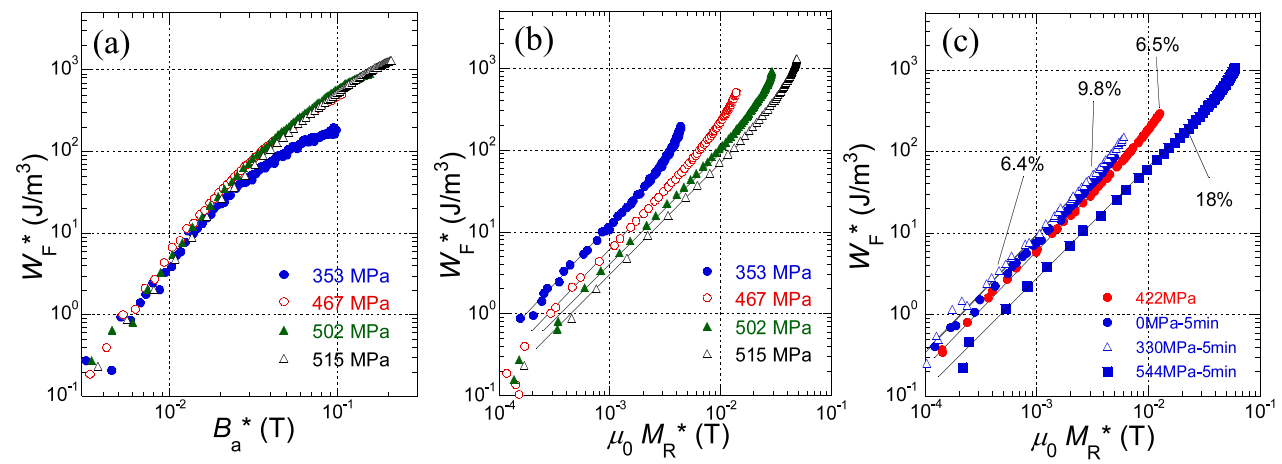

Figure 4. The relationship (a) between hysteresis loss $\left(W_{\mathrm{F}}^{*}\right)$ and maximum flux density $\left(B_{\mathrm{a}}{ }^{*}\right)$ and (b) between $W_{\mathrm{F}}{ }^{*}$ and remanence $\left(M_{\mathrm{R}}^{*}\right)$ plotted on a double logarithmic scale before and after plastic deformation. (c) The relationship between $W_{\mathrm{F}}{ }^{*}$ and $M_{\mathrm{R}}{ }^{*}$ plotted on a double logarithmic plot before and after cooling at $77 \mathrm{~K}$ for $5 \mathrm{~min}$. In (c), data obtained after cooling is shown as blue symbols. The number denoted in (c) is the volume fraction of $\alpha^{\prime}$-martensites. The solid lines in $(\mathbf{b}, \mathbf{c})$ denote the least-squares fitting lines.
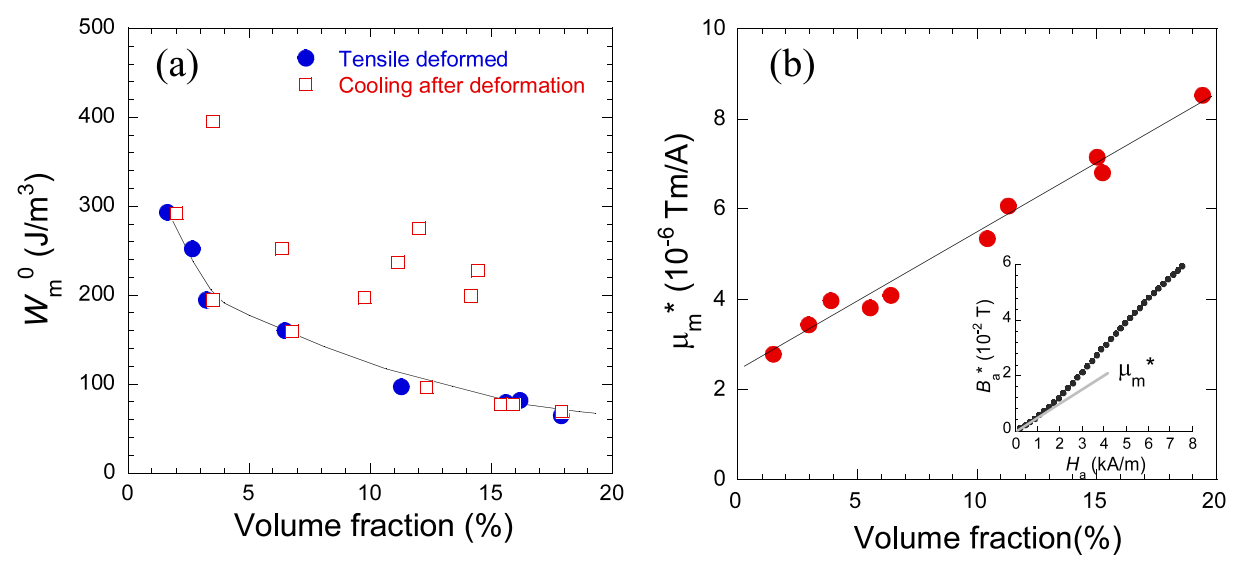

Figure 5. (a) $W_{\mathrm{m}}{ }^{0}$ as a function of the volume fraction of $\alpha^{\prime}$-martensites. The solid and open symbols denote the data before and after cooling at $77 \mathrm{~K}$ for $5 \mathrm{~min}$. (b) Minor-loop permeability $\left(\mu_{\mathrm{m}}{ }^{*}\right)$ as a function of volume fraction. The inset shows $B_{\mathrm{a}}{ }^{*}$ as a function of $H_{\mathrm{a}} \cdot \mu_{\mathrm{m}}{ }^{*}$ was obtained from a linear part of the $B_{\mathrm{a}}{ }^{*}-H_{\mathrm{a}}$ curve below $\left(H_{\mathrm{a}}=1 \mathrm{kA} / \mathrm{m}\right)$. Here, the step of field increase was about $100 \mathrm{~A} / \mathrm{m}$, therefore, approximately six minor loops were measured to determine $\mu_{\mathrm{m}}{ }^{*}$; this number can be further reduced if the field step is increased. Note that the number of data points obtained before and after cooling in (a) is different because minor-loop data for low volume fractions $(<2 \%)$ obtained before cooling was not analyzed.

The minor-loop coefficient $\left(W_{\mathrm{m}}{ }^{0}\right)$ is a structure-sensitive magnetic property similar to the $W_{\mathrm{F}}{ }^{0}$ obtained from the Steinmetz law, as well as coercivity. $W_{\mathrm{m}}{ }^{0}$ provides useful information about the size of $\alpha^{\prime}$-martensite particles, but it does not depend on the volume fraction of $\alpha^{\prime}$-martensites itself when the size is same for all the particles. This is because both $W_{\mathrm{F}}{ }^{*}$ and $B_{\mathrm{R}}{ }^{*}$ are parameters 
that depend on their volume fraction [6] and the scaling treatment of Equation (2) makes $W_{\mathrm{m}}{ }^{0}$ independent. Nevertheless, the volume fraction of $\alpha^{\prime}$-martensites can also be estimated from a set of minor hysteresis loops.

Figure $5 \mathrm{~b}$ shows minor-loop initial permeability $\left(\mu_{\mathrm{m}}{ }^{*}\right)$ as a function of volume fraction. Here, $\mu_{\mathrm{m}}{ }^{*}$ was obtained from a linear part of $B_{\mathrm{a}}{ }^{*}-H_{\mathrm{a}}$ curves below $\left(H_{\mathrm{a}}=1 \mathrm{kA} / \mathrm{m}\right)$, as shown in the inset of Figure $5 \mathrm{~b}$. It is clearly seen that $\mu_{\mathrm{m}}{ }^{*}$ is in linear proportion to the volume fraction. By measuring $\mu_{\mathrm{m}}{ }^{*}$ in addition to $W_{\mathrm{m}}{ }^{0}$, it is possible to obtain useful information on $\alpha^{\prime}$-martensites, such as the volume fraction and the morphology.

When the samples were subsequently cooled at $77 \mathrm{~K}$ (which is well below the martensitic transformation temperature), additional $\alpha^{\prime}$-martensite clusters with largely different shape are spontaneously formed (Figure 6). For samples with a volume fraction larger than $2 \%$, changes in volume fraction due to cooling were negligibly small; however, a large increase of the volume fraction up to $10-15 \%$ after cooling was detected in samples with a volume fraction below $0.2 \%$ (Figure 1a). This is due to the fact that small amounts of prestress facilitate the spontaneous formation of martensites, whereas the increasing dislocation density diminishes formation [5]. As shown in Figure $4 \mathrm{c}$, the relationship between $W_{\mathrm{F}}{ }^{*}$ and $M_{\mathrm{R}}{ }^{*}$ was found to follow the power law even in cooled samples. The least-squares fits to Equation (2) yielded an exponent of approximately 1.4; this is consistent with what was obtained before cooling. This strongly indicates that the scaling power law of Equation (2) universally holds true, and it does so independently from the morphology of $\alpha^{\prime}$-martensites and the volume fraction.

(a)

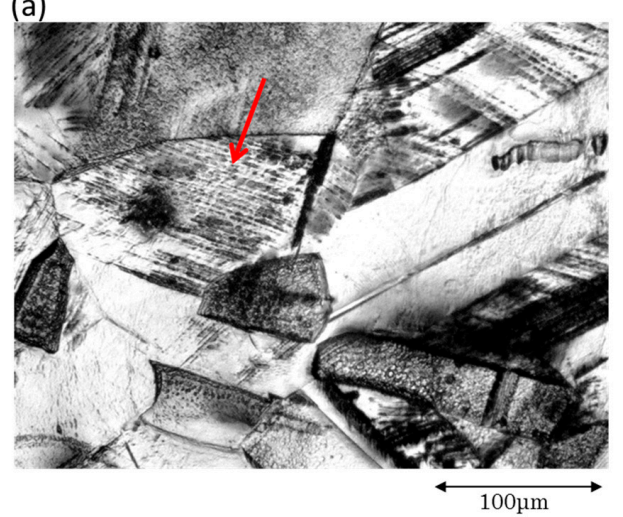

(b)

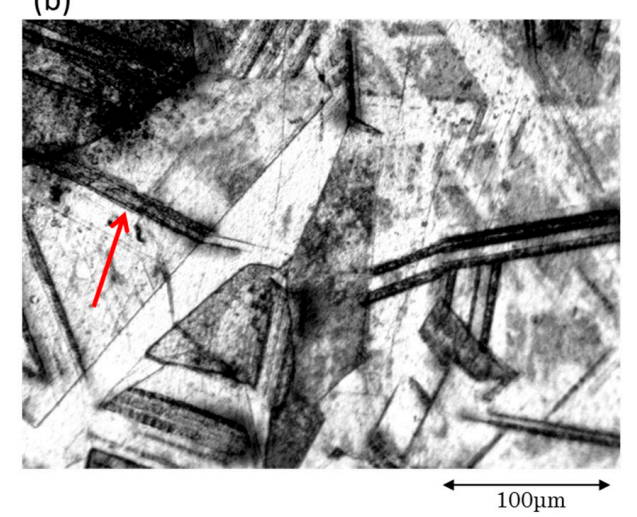

Figure 6. Optical microscope images of SUS304 stainless steels (a) after plastic deformation with a stress of $330 \mathrm{MPa}$ (volume fraction: $2.7 \%$ ) and (b) after cooling to $77 \mathrm{~K}$ for $5 \mathrm{~min}$ without pre-deformation (volume fraction: $9.8 \%$ ). Dark areas indicate martensite phases, which are colored by small magnetic particles approximately $10 \mathrm{~nm}$ in size.

In Figure $5 \mathrm{a}$, the coefficient as a function of volume fraction, obtained after cooling, is given. One can see that the coefficient exhibits a higher value compared to that obtained before cooling with the same volume fraction. This is a contrast to the behavior of coercivity shown in Figure $1 \mathrm{~b}$ where coercivity is reduced after cooling. Considering that the cooling process produces large martensites with the shape of long plates while deformation induces the formation of small martensites with the needle shape evident in Figure $6[4,10]$, the higher value of the coefficient for cooled samples may reflect the change in shape of $\alpha^{\prime}$-martensites due to cooling. If the shape is changed from needles to long plates, the demagnetizing field will increase, and remanence will reduce. Since $W_{\mathrm{m}}{ }^{0}$ was obtained from the $W_{\mathrm{F}}{ }^{*}-M_{\mathrm{R}}{ }^{*}$ curve, the reduction of remanence significantly shifts the curve upwards, as seen in Figure $4 \mathrm{~b}$; this results in an increase of $W_{\mathrm{m}}{ }^{0}$. If this effect is more pronounced compared to the reduced pinning effect caused by an increase in $\alpha^{\prime}$-martensite size after cooling, an increase of $W_{\mathrm{m}}{ }^{0}$ and a decrease of coercivity $\left(H_{\mathrm{c}}\right)$ after cooling could be experimentally observed. 


\section{Duplex Stainless Steel}

With recent requirements of combined mechanical performances (such as formability, high strength, and corrosion resistance), steels with a multi-phase microstructure like transformation-induced plasticity steels, dual phase steels, and duplex stainless steels have been widely used. In particular, duplex stainless steels have nearly equal amounts of magnetically soft ferrite $(\alpha)$ and non-magnetic $\gamma$ phases. A magnetically hard $\alpha^{\prime}$-martensite phase could be additionally formed in an austenitic matrix under plastic strain. Previous investigations demonstrated the usability of a magnetic method for duplex stainless steels which have been subjected to cold rolling or isothermal heat treatment [13-17]. In the case of cold-rolled steels, a good relationship between hysteresis properties (such as saturation magnetization, coercivity, and magnetic permeability) and the volume fraction of the deformed-induced $\alpha^{\prime}$-martensite phase was suggested. However, the relationship with minor-loop properties was not studied in detail; this may be useful for NDE owing to a low measurement field. Here, we have examined magnetic minor hysteresis loops for plastically-deformed duplex stainless steels to determine the applicability of the hysteresis scaling method to NDE of duplex stainless steels [18]. In this paper, particular attention was given to the dependence of minor-loop properties on the angle between the magnetizing and rolling direction.

\subsection{Materials and Experiments}

Plates of commercial SUS 329J4L duplex stainless steel with a thickness of $3 \mathrm{~mm}$ were used. The chemical composition for the steel and each phase (determined using a field emission electron probe microanalyzer) are listed in Table 2 . Here, the carbon content was determined with a carbon-sulfur combustion analyzer. Steel plates were cold-rolled to up to $90 \%$ rolling reduction (RD) in thickness direction, and several plates with different RD were prepared.

Figure 7a shows optical microscope images of the longitudinal section view for the $\mathrm{RD}=0 \%$ sample. Dark and light gray regions respectively represent $\alpha$ and $\gamma$ phases. This steel consists of both ferromagnetic $\alpha$ and non-magnetic $\gamma$ phases. Image analysis for different locations of specimens shows that the volume fraction of $\alpha$ and $\gamma$ phases in the present steels is $60 \pm 3 \%$ and $40 \pm 3 \%$, respectively. For the $\mathrm{RD}=0 \%$ sample, the $\gamma$ phase is homogeneously distributed like an island, and the thickness of both phases is reduced after cold rolling, (Figure 7b,c). Measurements showed that saturation magnetization is almost constant for a RD of up to $90 \%$, indicating that no martensitic phase transformation in the $\gamma$ phase takes place up to $\mathrm{RD}=90 \%$.

Table 2. Chemical composition of SUS329J4L steel and its constituent phases (wt. \%).

\begin{tabular}{cccccccc}
\hline Phase & $\mathbf{C}$ & $\mathbf{S i}$ & $\mathbf{M n}$ & $\mathbf{N i}$ & $\mathbf{C r}$ & Mo & Fe \\
\hline Steel & 0.015 & 0.72 & 0.78 & 5.55 & 25.70 & 2.89 & Bal. \\
$\alpha$ & - & 0.77 & 0.76 & 4.53 & 26.78 & 3.08 & Bal. \\
$\gamma$ & - & 0.66 & 0.81 & 6.41 & 24.35 & 2.28 & Bal. \\
\hline
\end{tabular}

(a) $\mathrm{RD}=0 \%$

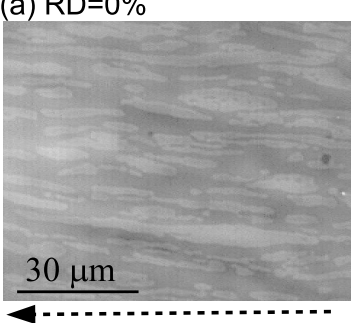

Rolling direction (b) $\mathrm{RD}=20 \%$

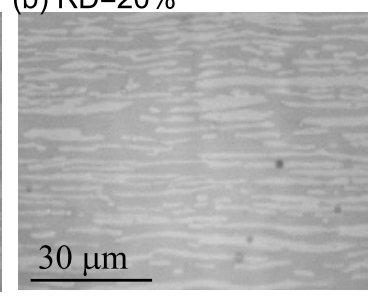

(c) $\mathrm{RD}=40 \%$

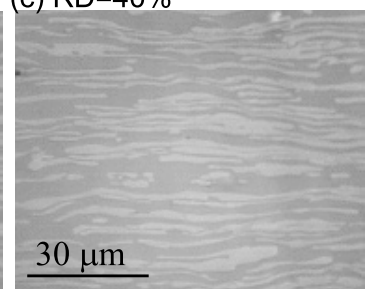

Figure 7. Optical microscope images, taken after (a) $0 \%$, (b) 20\%, and (c) $40 \%$ rolling reduction. Dark and light grays denote bcc ferrite $(\alpha)$ and fcc austenitic $(\gamma)$ phases, respectively. 
Minor $B$ - $H$ loops were measured with a flux meter for a disk sample. A U-shape iron yoke wound with 500-turn exciting and 200-turn pickup coils was attached to the disk to form a magnetically closed circuit. The direction of the long axis of the yoke with respect to the rolling direction, $\theta$, was changed to investigate the dependence of the minor-loop properties angle between the magnetizing and rolling directions. Our measurement setup is almost the same as that described in Section 2.1. The field sweep rate $(d H / d t)$ was set to $13 \mathrm{kA} / \mathrm{m} \cdot \mathrm{s}$, which was low enough to neglect the eddy current effect. By changing the amplitude of a cyclic magnetic field to up to $20 \mathrm{kA} / \mathrm{m}$ step-by-step, a set of minor $B-H$ loops was obtained.

\subsection{Results and Discussion}

Figure 8 shows a set of minor $B-H$ loops before and after cold rolling. With increasing reduction, loop width and remanent flux density both increase with each minor loop. For minor loops with the same rolling reduction, no pronounced angle dependence was observed. To examine the hysteresis scaling behavior of minor loops, parameters of each minor $B-H$ loop with different field amplitudes were determined.
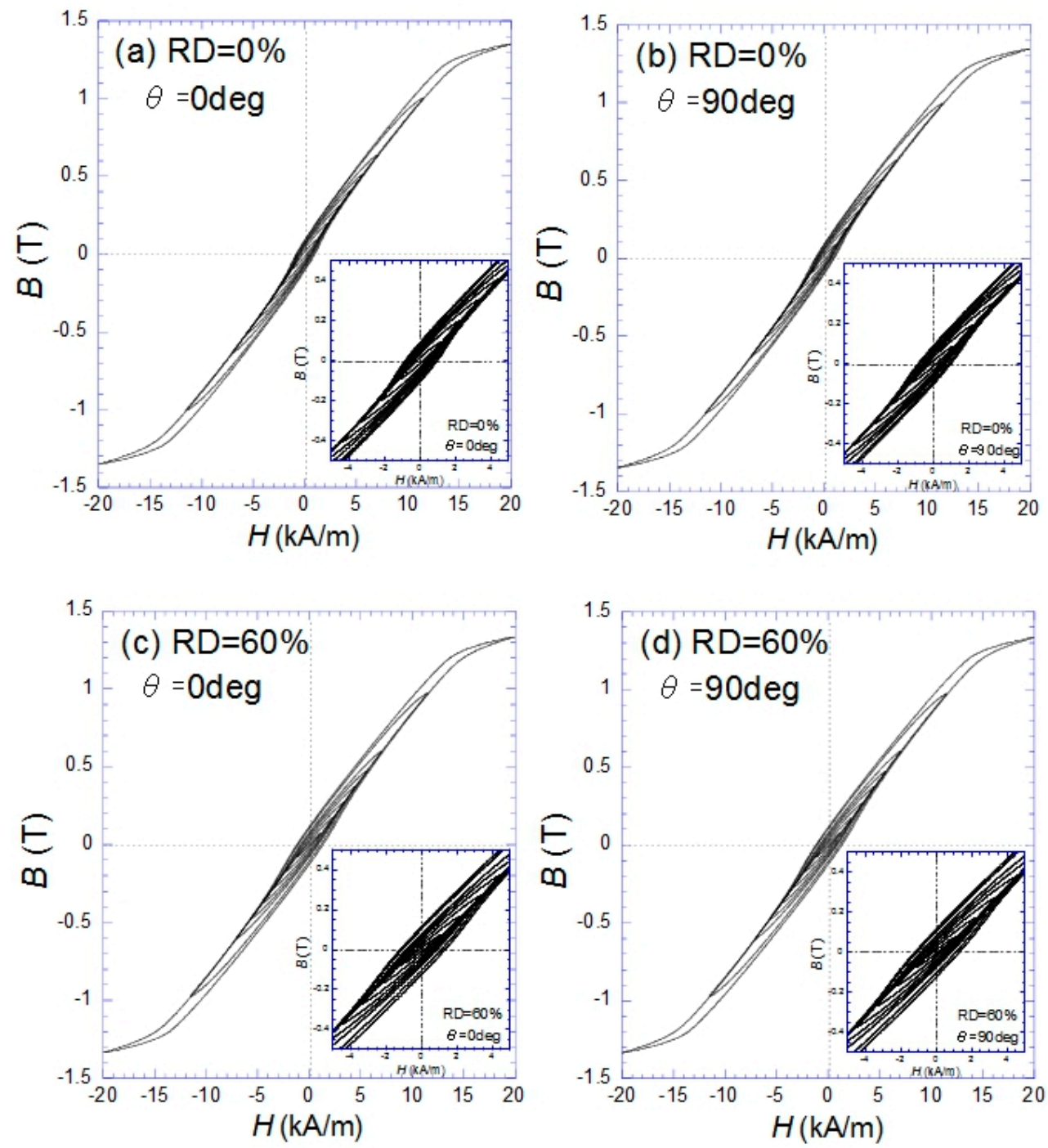

Figure 8. A set of minor $B(H)$ loops before and after cold rolling. The angle $\theta$ represents the rolling direction; (a) $\mathrm{RD}=0 \%$ and $\theta=0^{\circ}$, (b) $\mathrm{RD}=0 \%$ and $\theta=90^{\circ}$, (c) $\mathrm{RD}=60 \%$ and $\theta=0^{\circ}$, and (d) $\mathrm{RD}=60 \%$ and $\theta=90^{\circ}$. 
Figure 9a,b show relationships between hysteresis loss and maximum flux density, before and after cold rolling for different angles, plotted in a double logarithmic scale. For comparison, the slope of 1.5 is given. When $B_{\mathrm{a}}{ }^{*}$ is above approximately $3 \times 10^{-1} \mathrm{~T}$, the curves for all values of $\theta$ (obtained before and after rolling reduction) exhibit a linearity; this suggests that a power law exists between $W_{\mathrm{F}}{ }^{*}$ and $B_{\mathrm{a}}{ }^{*}$. For the relationship between $W_{\mathrm{F}}{ }^{*}$ and $B_{\mathrm{R}}{ }^{*}$, a linearity was also observed from the low to medium $B_{R}{ }^{*}$ range (at less than approximately $3 \times 10^{-2} \mathrm{~T}$ ) for all reductions and values of $\theta$ (Figure $9 \mathrm{c}, \mathrm{d}$ ). This indicates a power law relationship between $W_{\mathrm{F}}{ }^{*}$ and $B_{\mathrm{R}}{ }^{*}$. Assuming Equations (1) and (2) for the $W_{\mathrm{F}}{ }^{*}-B_{\mathrm{a}}{ }^{*}$ and $W_{\mathrm{F}}{ }^{*}-B_{\mathrm{R}}{ }^{*}$ curves, respectively, exponents of $n_{\mathrm{F}}=1.48 \pm 0.02$ and $n_{\mathrm{m}}=1.34 \pm 0.05$ were obtained. Here, minor loops in the range of $H_{\mathrm{a}}$ from 3 to $12 \mathrm{kA} / \mathrm{m}$ were used to determine $W_{\mathrm{F}}{ }^{0}$, while for $W_{\mathrm{m}}{ }^{0}$, minor loops with $H_{\mathrm{a}}$ from $0.2 \mathrm{kA} / \mathrm{m}$ to $2 \mathrm{kA} / \mathrm{m}$ were used. The exponent values are almost the same as previously reported values for ferromagnetic materials [2,3], indicating the universality of the hysteresis scaling law for minor loops.
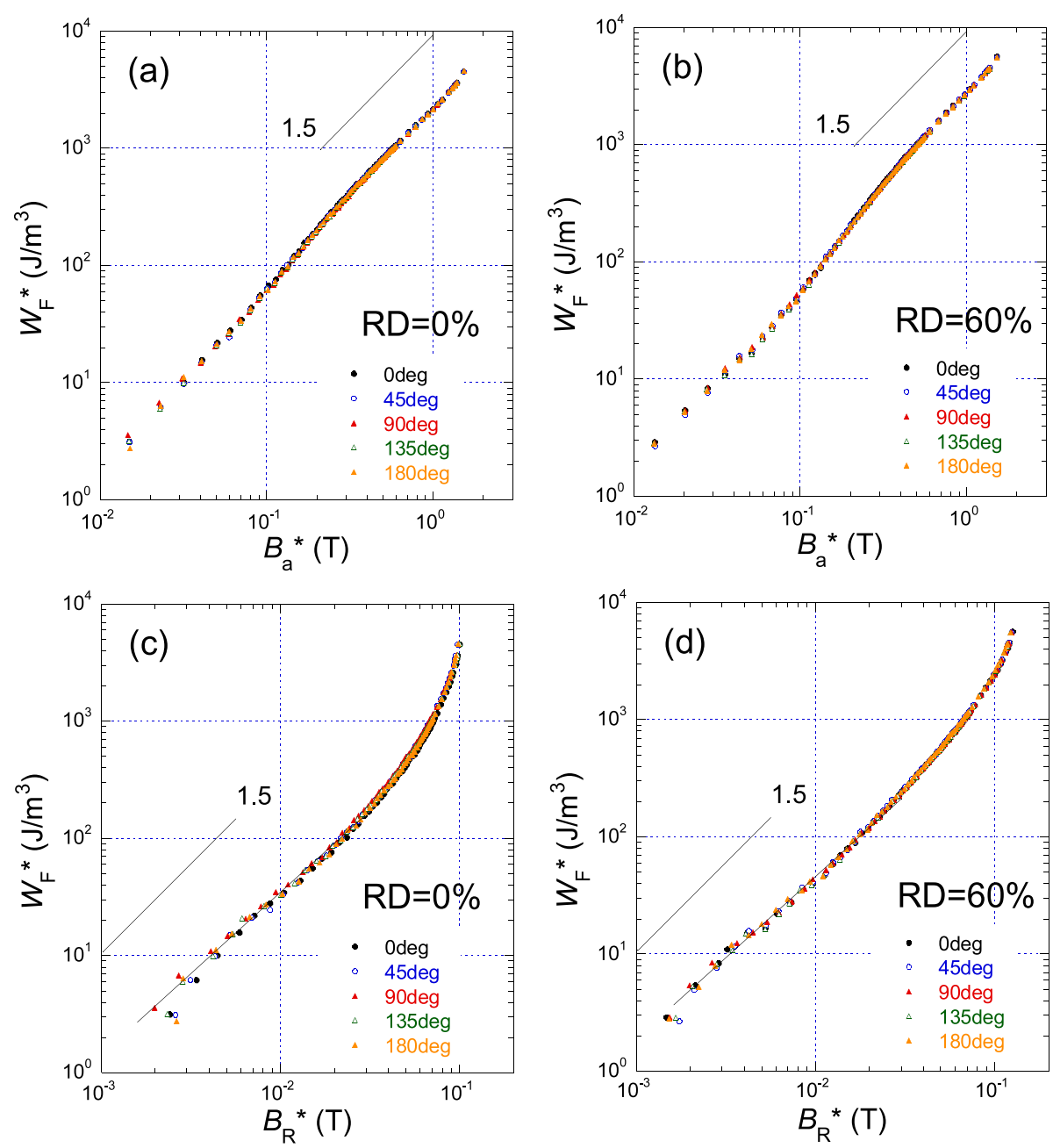

Figure 9. Double logarithmic plots of the relationship between $W_{\mathrm{F}}{ }^{*}$ and $B_{\mathrm{a}}{ }^{*}$ at $(\mathbf{a}) \mathrm{RD}=0 \%$ and (b) RD $=60 \%$, and the relationship between $W_{\mathrm{F}}{ }^{*}$ and $B_{\mathrm{R}}{ }^{*}$ at (c) $\mathrm{RD}=0 \%$ and (d) $\mathrm{RD}=60 \%$. For each plot, relations obtained with different values of $\theta$ are given.

Figure 10a,b show the $\theta$-dependence of minor-loop coefficients $W_{\mathrm{F}}{ }^{0}$ and $W_{\mathrm{m}}{ }^{0}$, respectively, taken at different levels of rolling reduction. As shown in Figure $10 \mathrm{a}, W_{\mathrm{F}}{ }^{0}$ is nearly isotropic in shape; no pronounced $\theta$-dependence was observed for all the reductions. As the rolling reduction increases, $W_{\mathrm{F}}{ }^{0}$ monotonically increases. This increase can be due to an increase of dislocation density with rolling reduction [18]. On the other hand, the $\theta$-dependence of $W_{\mathrm{m}}{ }^{0}$ is slightly anisotropic in 
shape; the anisotropy seems to depend on the level of rolling reduction. At low rolling reduction (less than approximately $10 \%$ ), $W_{\mathrm{m}}{ }^{0}$ maximizes when magnetizing direction is perpendicular to the rolling direction, or when $\theta$ is at approximately $90^{\circ}$ and $270^{\circ}$. As the rolling reduction increases, the $\theta$-dependence becomes gradually isotropic in shape; $W_{\mathrm{m}}{ }^{0}$ then exhibits the maximum value when $\theta$ is at approximately $0^{\circ}$ and $180^{\circ}$, at a rolling reduction above $30 \%$. This anisotropy in $W_{\mathrm{m}}{ }^{0}$ is opposite to that observed at a low rolling reduction. The magnitude of $W_{\mathrm{m}}{ }^{0}$ exhibits a steady increase with rolling reduction, reflecting an increase of dislocation density.

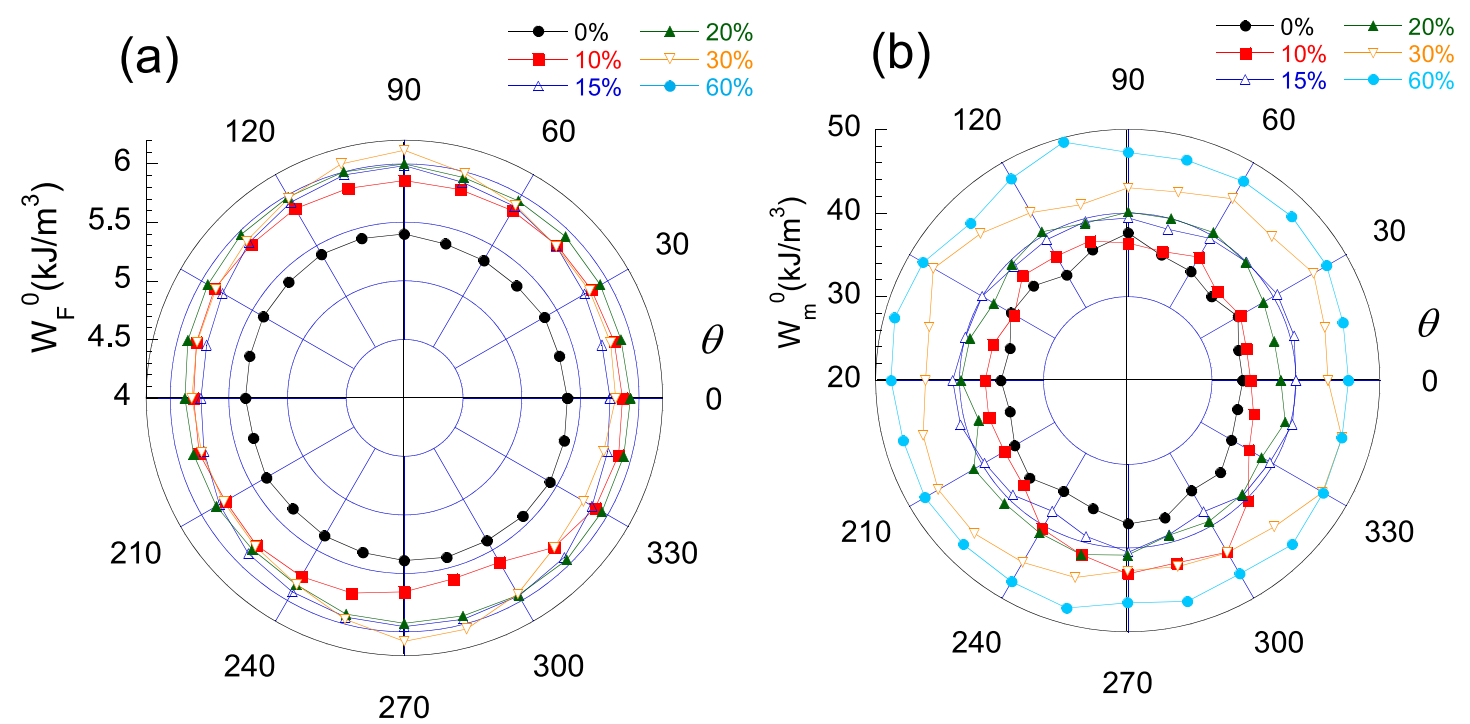

Figure 10. The $\theta$-dependence of $(\mathbf{a}) W_{\mathrm{F}}^{0}$ and (b) $W_{\mathrm{m}}{ }^{0}$ obtained at different levels of rolling reduction.

For almost all cases, the coefficient is proportionally related to defect density. However, as discussed in Section 2.2, $W_{\mathrm{m}}{ }^{0}$ can reflect the morphology of ferromagnetic regions, i.e., their size and shape. Since the morphology of the ferromagnetic $\alpha$ phase changes with rolling reduction in duplex stainless steels, shape may influence the behavior of $W_{\mathrm{m}}{ }^{0}$. For $\mathrm{RD}=0 \%$, the sample contains $\gamma$ phase particles with a nearly prolate spheroid shape, as shown in Figure 7a; a large number of magnetic poles may be produced at the $\alpha-\gamma$ interface. This yields a demagnetizing field and reduces $B_{\mathrm{R}}{ }^{*}$ for each minor loop. The reduction of $B_{\mathrm{R}}{ }^{*}$ will maximize when the magnetizing direction is perpendicular to rolling reduction (i.e., perpendicular to the long axis of $\gamma$ phases). This results in a shift of the $W_{\mathrm{F}}{ }^{*}-B_{\mathrm{R}}{ }^{*}$ curve toward a lower $B_{\mathrm{R}}{ }^{*}$, leading to a large value of $W_{\mathrm{m}}{ }^{0}$. The demagnetizing field-which depends on the angle of magnetizing direction in relation to rolling reduction-may be responsible for the anisotropy in the $\theta$-dependence of $W_{\mathrm{m}}{ }^{0}$ at low levels of rolling reduction.

As the rolling reduction increases, dislocation density increases; this accompanies the development of texture. According to X-ray texture measurements for SUS 329J4L duplex stainless steels, the $\alpha$ phase exhibits a typical cold-rolling texture, such as $\{100\}<011>$ or $\{211\}<011>$, although the fraction of the types of texture depends on the level of rolling reduction $[19,20]$. Since the bcc $\alpha$ ferrite phase has a magnetic anisotropy axis of $\langle 100\rangle$, such change of texture should influence the magnetization process for each $\theta$. Moreover, the morphology of the $\gamma$ phase also changes with rolling reduction. Therefore, the $\theta$-dependence of the demagnetizing field also changes. At present, the observed change of anisotropy in the $\theta$-dependence of $W_{\mathrm{m}}{ }^{0}$ with increasing rolling reduction is not fully understood. Nevertheless, the combined effects of changes in texture and morphology of the $\alpha$ phase may yield the observed $\theta$-dependence of $W_{\mathrm{m}}{ }^{0}$ shown in Figure 10b. Further examinations using the electron backscatter diffraction (EBSD) technique is planned. 


\section{Conclusions}

The present study demonstrates that a scaling power law between hysteresis loss and remanence of minor hysteresis loops universally holds true for ferromagnetic $\alpha^{\prime}$-martensites formed in an austenitic stainless steel, as well as duplex stainless steel in which $\alpha$ and $\gamma$ phases coexist. In both types of steel, the observed exponent ranges from approximately 1.3 to 1.4, which is the same as that of ferromagnetic steels. While the coefficient of the law for austenitic stainless steels is sensitive to the morphology of $\alpha^{\prime}$-martensite in a $\gamma$ matrix, it reflects the morphology of $\alpha$ and $\gamma$ phases for duplex stainless steels. Furthermore, in both cases, the coefficient can be obtained with low magnetic fields below $4 \mathrm{kA} / \mathrm{m}$, which is about a hundredth of the field required for saturation measurements. This magnetic method using minor hysteresis loops can, therefore, be a possible NDE technique for the in situ integrity assessment of stainless steel components where compact, portable, and light devices are highly preferable.

As in the case of major-loop coercivity, the minor-loop coefficient has a good relationship with defect density; therefore, the minor loop method can be used for "quantitative" NDE. However, the method may be limited for a simple case such as ferromagnetic steels subjected to tensile deformation in which only one type of defect (dislocation in this case) mainly contributes to the magnetic property. The minor-loop coefficient is a magnetic property averaging over the whole sample, therefore, it is difficult to quantitatively speculate what microstructural change takes place when various types of microstructural changes occur simultaneously; one example is nuclear reactor pressure vessel steels subjected to neutron irradiation, where magnetic hardening due to the formation of nanoscale precipitates and softening due to recovery compete [4].

For practical application of the minor-loop method, magnetic yoke should be used. In this case, the condition of contactness between the yoke and sample surface influences shape of minor loops as in the case of other magnetic methods. Among the minor-loop parameters, remanent flux density $B_{\mathrm{R}}{ }^{*}$ is largely affected and reduced for poor contactness. Therefore, the minor-loop coefficient $W_{\mathrm{m}}{ }^{0}$ is more sensitive to surface conditions as compared to $W_{\mathrm{F}}{ }^{0}$. For practical applications, and $W_{\mathrm{F}}{ }^{0}$ may be used to obtain consistent results. However, for good contactness, $W_{\mathrm{m}}{ }^{0}$ can be a very useful NDE parameter because of the extremely lower measurement field than that for $W_{\mathrm{F}}{ }^{0}$.

Acknowledgments: We thank Koji Sasaki, Michihiro Ito and Katsuhiko Nonaka for their assistance with the chemical analysis. We thank Yasuhiro Kamada for valuable discussions.

Author Contributions: Satoru Kobayashi designed and performed measurements, analyzed data, and wrote the paper; Kodai Miura partly contributed to the magnetic measurements and optical microscopy for SUS304 austenitic stainless steels; Kodai Miura and Yuki Narita partly contributed to the magnetic measurements and optical microscopy for duplex stainless steels; and Seiki Takahashi contributed to interpretation of results of minor-loop measurements.

Conflicts of Interest: The authors declare no conflict of interest.

\section{References}

1. Steinmetz, C.P. On the law of hysteresis. IEEE Proc. 1964, 72, 197-221. [CrossRef]

2. Takahashi, S.; Kobayashi, S.; Kikuchi, H.; Kamada, Y. Relationship between mechanical and magnetic properties in cold rolled low carbon steel. J. Appl. Phys. 2006, 100, 113908. [CrossRef]

3. Kobayashi, S.; Takahashi, S.; Shishido, T.; Kamada, Y.; Kikuchi, H. Low-field magnetic characterization of ferromagnets using a minor-loop scaling law. J. Appl. Phys. 2010, 107, 023908. [CrossRef]

4. Kobayashi, S.; Yamamoto, T.; Klingensmith, D.; Odette, G.R.; Kikuchi, H.; Kamada, Y. Magnetic evaluation of irradiation hardening in A533B reactor pressure vessel steels: Magnetic hysteresis measurements and the model analysis. J. Nucl. Mater. 2012, 422, 158-162. [CrossRef]

5. Lagneborg, R. The Martensite Transformation in 18\%Cr-8\%Ni Steels. Acta Metall. 1964, 12, $823-843$. [CrossRef] 
6. Mitra, A.; Srivastava, P.K.; De, P.K.; Bhattacharya, D.K.; Jiles, D.C. Ferromagnetic Properties of Deformation-Induced Martensite Transformation in AISI 304 Stainless Steel. Metall. Mater. Trans. A 2004, 35A, 599-605.

7. Meszaros, I.; Prohaszka, J. Magnetic investigation of the effect of $\alpha^{\prime}$-martensite on the properties of austenitic stainless steel. J. Mater. Proc. Technol. 2005, 161, 162-168. [CrossRef]

8. Ding, J.; Huang, H.; McCormick, P.G.; Street, R. Magnetic properties of martensite-austenite mixtures in mechanically milled 304 stainless steel. J. Mag. Magn. Mater. 1995, 139, 109-114. [CrossRef]

9. Tavares, S.S.M.; da Silva, M.R.; Neto, J.M.; Miraglia, S.; Fruchart, D. Ferromagnetic properties of cold rolled AISI 304L steel. J. Magn. Magn. Mater. 2002, 242-245, 1391-1394. [CrossRef]

10. Zhang, L.; Takahashi, S.; Kamada, Y. Quench-Induced Martensitic Transformation in Austenitic Stainless Steel after Tensile Deformation at Elevated Temperature. Scr. Mater. 2007, 57, 711-714. [CrossRef]

11. Kobayashi, S.; Saito, A.; Takahashi, S.; Kamada, Y.; Kikuchi, H. Characterization of strain-induced martensite phase in austenitic stainless steel using a magnetic minor-loop scaling relation. Appl. Phys. Lett. 2008, 92, 182508. [CrossRef]

12. Kobayashi, S.; Kikuchi, N.; Takahashi, S.; Kamada, Y.; Kikuchi, H. Magnetic properties of $\alpha^{\prime}$ martensite in austenitic stainless steel studied by a minor-loop scaling law. J. Appl. Phys. 2010, 108, 043904. [CrossRef]

13. Bassani, P.; Breda, M.; Brunelli, K.; Meszaros, I.; Passaretti, F.; Zanellato, M.; Calliari, I. Characterization of a cold-rolled 2101 lean duplex stainless steel. Microsc. Microanal. 2013, 19, 988-995. [CrossRef] [PubMed]

14. Tavares, S.S.M.; da Silva, M.R.; Pardal, J.M.; Abreu, H.F.G.; Gomes, A.M. Microstructural changes produced by plastic deformation in the UNS S31803 duplex stainless steel. J. Mater. Process. Technol. 2006, 180, 318-322. [CrossRef]

15. Baldo, S.; Meszaros, I. Effect of cold rolling on microstructure and magnetic properties in a metastable lean duplex stainless steel. J. Mater. Sci. 2010, 45, 5339-5346. [CrossRef]

16. Meszaros, I.; Szabo, P.J. Complex magnetic and microstructural investigation of duplex stainless steel. NDT E Int. 2005, 38, 517-521. [CrossRef]

17. Tavares, S.S.M.; Pardal, J.M.; Guerreiro, J.L.; Gomes, A.M.; da Silva, M.R. Magnetic detection of sigma phase in duplex stainless steel UNS S31803. J. Magn. Magn. Mater. 2010, 322, L29-L33. [CrossRef]

18. Kobayashi, S.; Murakami, T.; Narita, Y.; Miura, K.; Kamada, Y. Effects of cold rolling on magnetic minor hysteresis loops of duplex stainless steels. Philos. Mag. 2017, 97, 419-430. [CrossRef]

19. Hamada, J.; Ono, N. Effect of Microstructure before Cold Rolling on Texture and Formability of Duplex Stainless Steel Sheet. Mater. Trans. 2010, 51, 635-643. [CrossRef]

20. Hamada, J.; Inoue, H. Texture and Planar Anisotropy of $r$-Value in Duplex Stainless Steel Sheet. Mater. Trans. 2010, 51, 644-651. [CrossRef]

(C) 2017 by the authors. Licensee MDPI, Basel, Switzerland. This article is an open access article distributed under the terms and conditions of the Creative Commons Attribution (CC BY) license (http:// creativecommons.org/licenses/by/4.0/). 